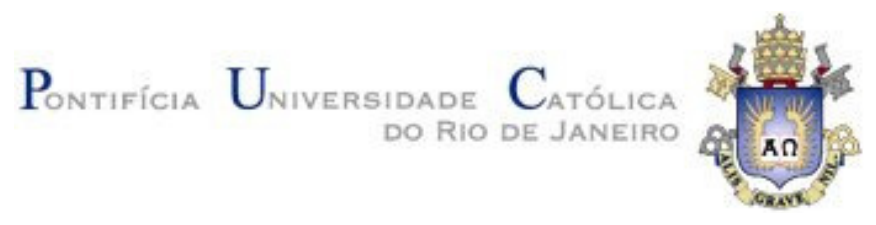

Maurício Serrano

\title{
Desenvolvimento Intencional de Software Transparente Baseado em Argumentação
}

Tese de Doutorado

Tese apresentada ao Programa de Pós-Graduação em Informática do Departamento de Informática da PUC-Rio como parte dos requisitos parciais para obtenção do título de Doutor em Informática.

Orientador: Prof. Julio Cesar Sampaio do Prado Leite

Rio de Janeiro 


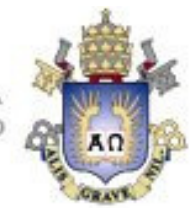

Maurício Serrano

\section{Desenvolvimento Intencional de Software Transparente Baseado em Argumentação}

Tese apresentada como requisito parcial para obtenção do grau de Doutor pelo Programa de Pós-graduação em Informática do Departamento de Informática do Centro Técnico Científico da PUC-Rio. Aprovada pela Comissão Examinadora abaixo assinada.

Prof. Julio Cesar Sampaio do Prado Leite Orientador Departamento de Informática - PUC-Rio

Prof. Carlos José Pereira de Lucena Departamento de Informática - PUC-Rio

Prof(a). Simone Diniz Junqueira Barbosa

Departamento de Informática - PUC-Rio

Prof. Sérgio Donizetti Zorzo Departamento de Computação - UFSCar

Prof(a). Claudia Cappelli Aló

Departamento de Informática Aplicada - UNIRIO

Prof. José Eugenio Leal Coordenador Setorial do Centro Técnico Científico - PUC-Rio

Rio de Janeiro, 23 de Agosto de 2011. 
Todos os direitos reservados. É proibida a reprodução total ou parcial do trabalho sem autorização do autor, do orientador e da universidade.

\section{Maurício Serrano}

Graduou-se em Engenharia de Computação (2001) e obteve o título de mestre em Ciência da Computação (2003) na Universidade Federal de São Carlos (UFSCar) - financiado pela CAPES (Bolsista por Mérito). Trabalhou no Projeto Genoma do Camarão (2003-2004) - financiado pela FAPESP. Durante seu Doutorado - financiado pela FAPESP/CAPES (Bolsista por Mérito) - na Pontifícia Universidade Católica do Rio de Janeiro (PUC-Rio), sob a orientação do Professor Julio Cesar Sampaio do Prado Leite, teve um período de cooperação na University of Toronto (UofT), sob a orientação do Professor John Mylopoulos. Tem experiência em Engenharia de Software e Engenharia de Requisitos com foco, principalmente, nos tópicos: Orientação à Meta, Sistemas Multi-Agentes Intencionais e Transparência de Software.

Ficha Catalográfica

Serrano, Maurício

Desenvolvimento intencional de software transparente baseado em argumentação / Maurício Serrano; orientador: Julio Cesar Sampaio do Prado Leite. - 2011.

xviii., $283 \mathrm{f}$ : il. ; $30 \mathrm{~cm}$.

Tese (Doutorado em Informática) - Pontifícia Universidade Católica do Rio de Janeiro, Rio de Janeiro, 2011.

Inclui bibliografia.

1. Informática - Teses. 2. Transparência de software. 3. Requisitos de transparência. 4. Argumentação. 5. Validação relativa. 6. Rastreabilidade. 7. Máquina de raciocínio. 8. Modelos intencionais. 9. Sistemas multiagentes intencionais. I. Leite, Julio Cesar Sampaio do Prado. II. Pontifícia Universidade Católica do Rio de Janeiro. Departamento de Informática. III. Título.

CDD: 004 
Para Minha Amada Família: Minha Esposa Milene, Minha Mãe Maria Elizabeth, Meu Pai Edson (in memoriam), Minhas Irmãs Adriana e Mariana, Minha Sogra Elizabeth, Meu Sogro Antonio e Minha Cunhada Gabriela. 


\section{Agradecimentos}

Meus mais especiais agradecimentos ao meu Orientador, Professor Julio Cesar Sampaio do Prado Leite, pela dedicação e pelo apoio ao longo de toda a pesquisa.

Meu muito obrigado a minha querida família pelo carinho, pelos conselhos e pela paciência.

Agradeço aos Professores John Mylopoulos e Eric Yu pela maravilhosa experiência na University of Toronto, Canadá.

Agradeço ainda ao Professor Carlos José Pereira de Lucena e à Professora Simone Diniz Junqueira Barbosa pelas reuniões e contribuições ao longo de toda a pesquisa.

Agradecimentos carinhosos aos colegas do Grupo de Transparência de Software e do Laboratório de Engenharia de Software (LES) da PUC-Rio.

Meu muito obrigado ao corpo docente e aos funcionários da PUC-Rio pela atenção e pela prontidão em ajudar.

Meus sinceros agradecimentos aos membros da banca, Professores e Professoras, pela participação na defesa da tese de doutorado.

Finalmente, agradeço à agência de fomento CNPq pelo apoio financeiro. 


\section{Resumo}

Serrano, Maurício; Leite, Julio Cesar Sampaio do Prado. Desenvolvimento Intencional de Software Transparente Baseado em Argumentação. Rio de Janeiro, 2011. 283p. Tese de Doutorado - Departamento de Informática, Pontifícia Universidade Católica do Rio de Janeiro.

Transparência é um critério de qualidade crítico para sociedades democráticas modernas. Como o software permeia a sociedade, a transparência se tornou uma preocupação para softwares operando em domínios públicos, sejam eles eGovernment, eCommerce ou softwares sociais. Dessa forma, a transparência de software está se tornando um critério de qualidade que demanda mais atenção dos desenvolvedores de software. Requisitos de transparência em um sistema de software estão relacionados a requisitos não-funcionais, como disponibilidade, usabilidade, informatividade, entendimento e auditabilidade. Entretanto, requisitos de transparência são especialmente difíceis de serem validados devido à natureza subjetiva dos conceitos envolvidos. Essa tese propõe o desenvolvimento intencional de software transparente dirigido por requisitos de transparência. Os requisitos de transparência são elicitados com o apoio de um catálogo de padrões de requisitos, relativamente validados pelos interessados através do uso de argumentação e representados em modelos intencionais. Modelos intencionais são fundamentais para a transparência de software, uma vez que associam aos requisitos as metas e os critérios de qualidade esperados pelos interessados e que justificam as decisões tomadas. Um sistema exemplo foi implementado como um sistema multi-agentes intencional, ou seja, com agentes colaborativos que implementam o modelo Belief-Desire-Intention e que são capazes de raciocinar sobre metas e critérios de qualidade. Essa tese discute as questões importantes para o sucesso da nossa abordagem de desenvolvimento de software transparente, como: (i) rastreabilidade requisitos-código e código-requisitos; (ii) o uso de lógica nebulosa para desenvolver uma máquina de raciocínio para agentes intencionais; (iii) a aplicação de argumentação para a validação relativa de requisitos de transparência através da obtenção de um consenso entre os interessados; e (iv) pré-rastreabilidade colaborativa para modelos intencionais baseada nas interações sociais. Nossas idéias foram validadas através de estudos de caso em diferentes domínios, tal como computação ubíqua e aplicações Web. 


\section{Palavras-chave}

Transparência de Software; Requisitos de Transparência; Argumentação;

Validação Relativa; Rastreabilidade; Máquina de Raciocínio; Modelos Intencionais; Sistemas Multi-Agentes Intencionais. 


\section{Abstract}

Serrano, Maurício; Leite, Julio Cesar Sampaio do Prado. Intentional Development of Transparent Software Based on Argumentation. Rio de Janeiro, 2011. 283p. DSc Thesis - Departamento de Informática, Pontifícia Universidade Católica do Rio de Janeiro.

Transparency is a critical quality criterion to modern democratic societies. As software permeates society, transparency has become a concern to public domain software, as eGovernment, eCommerce or social software. Therefore, software transparency is becoming a quality criterion that demands more attention from software developers. In particular, transparency requirements of a software system are related to non-functional requirements, e.g. availability, usability, informativeness, understandability and auditability. However, transparency requirements are particularly difficult to validate due to the subjective nature of the involved concepts. This thesis proposes a transparency-requirements-driven intentional development of transparent software. Transparency requirements are elicited with the support of a requirements patterns catalog, relatively validated by the stakeholders through argumentation and represented on intentional models. Intentional models are fundamental to software transparency, as they associate goals and quality criteria expected by the stakeholders with the software requirements. The goals and quality criteria also justify the decisions made during software development. A system was implemented as an intentional multi-agents system, i.e., a system with collaborative agents that implement the Belief-DesireIntention model and that are capable of reasoning about goals and quality criteria. This thesis discusses important questions to the success of our approach to the development of transparent software, such as: (i) forward and backward traceability; (ii) a fuzzy-logic based reasoning engine for intentional agents; (iii) the application of an argumentation framework to relatively validate transparency requirements through stakeholders' multi-party agreement; and (iv) collaborative pre-traceability for intentional models based on social interactions. Our ideas were validated through case studies from different domains, such as ubiquitous computing and Web applications. 


\section{Keywords}

Software Transparency; Tranparency Requirements; Argumentation;

Relative Validation; Traceability; Reasoning Engine; Intentional Models; Intentional Multi-Agent Systems. 


\section{Sumário}

1. Introdução 20

1.1. Motivação 20

1.2. Caracterização do Problema 23

1.3. Enfoque da Solução 24

1.4. Organização da Tese 25

2. Conceitos e Apoio Tecnológico 26

2.1. Modelos Intencionais: Os Frameworks i e NFR 26

2.2. Transparência de Software 29

2.3. Sistemas Multi-Agentes Intencionais 30

2.4. O Framework Acceptability Evaluation 34

2.5. Considerações Finais 36

3. Anexação de Requisitos ao Código 38

3.1. Associações entre Abstrações dos Modelos i* e BDI e Código JADEX 40

3.2. Heurísticas Transformacionais de Desenho e de Implementação 43

3.3. Geração dos Rastros a partir das Heurísticas Transformacionais 53

3.4. Trabalhos Relacionados 54

3.5. Considerações Finais

4. Raciocínio sobre Metas Flexíveis em Tempo de Execução 56

4.1. O Simulador de Propagação 59

4.2. Máquina de Raciocínio Qualitativa 65

4.3. Trabalhos Relacionados 83

4.4. Considerações Finais $\quad 87$

5. Argumentação sobre Requisitos de Transparência 88

5.1. Representando Modelos Intencionais no Framework ACE 90

5.2. Condição de Aceitabilidade 96 
5.3. Captura dos Argumentos 99

5.4. Validação Relativa dos Requisitos de Transparência 101

5.5. Apoio Ferramental 111

5.6. Trabalhos Relacionados 115

5.7. Considerações Finais 116

6. Pré-Rastreabilidade 118

6.1. Visão Geral do Modelo ITrace 121

6.2. Representação Gráfica dos Modelos ITrace 123

6.3. Pré-Rastreabilidade Colaborativa 130

6.4. Trabalhos Relacionados 132

6.5. Considerações Finais 132

7. Abordagem Integrada para o Desenvolvimento de Software $\begin{array}{ll}\text { Transparente } & 134\end{array}$

7.1. Abordagem Proposta para Desenvolvimento Intencional Baseada em $\begin{array}{ll}\text { Argumentação } & 135\end{array}$

7.2. Estudo de Caso: Aplicação Web Lattes-Scholar 138

7.2.1. Atividade Argumentar 139

7.2.2. Atividade Desenhar 146

7.2.3. Atividade Implementar 150

7.2.4. Atividades Armazenar e Consultar 155

7.3. Considerações Finais 156

8. Avaliação da Abordagem Integrada 158

8.1. Avaliação Quantitativa da Aplicação da Abordagem 160

8.2. Avaliação Qualitativa da Aplicação da Abordagem 166

8.3. Avaliação da Abordagem Integrada por Construção 169

8.4. Análise das Versões Anteriores do Lattes-Scholar 176

8.5. Considerações Finais 180

9. Conclusão 181

9.1. Contextualização 181

9.2. Resumo 182 
9.3. Contribuições dos Trabalhos Pontuais

9.3.1. Anexação de Requisitos ao Código

9.3.2. Raciocínio sobre Metas Flexíveis em Tempo de Execução

9.3.3. Argumentação sobre Requisitos de Transparência

9.3.4. Pré-Rastreabilidade

9.4. Estudos de Caso para os Trabalhos Pontuais 189

9.5. Contribuições Relacionadas à Abordagem Integrada 190

9.6. Estudo de Caso para a Abordagem Integrada 191

9.7. Avaliação da Abordagem Integrada 194

9.8. Esclarecimento das Questões Levantadas na Introdução 195

9.9. Limitações 197

9.10. Trabalhos Futuros 198

Apêndice A. Exemplos de Uso do Lattes-Scholar 212

A.1 Processo Iniciado pelo Consumidor com o Nome do Pesquisador 212

A.2 Processo Iniciado pelo Consumidor com a URL do

Currículo Lattes do Pesquisador

Apêndice B. Código-Fonte da Máquina de Raciocínio Nebulosa para Metas Flexíveis

B.1 Código-fonte da Classe MetaPlan

B.2 Código-fonte da Classe Softgoal

B.3 Código-fonte da Classe LeafSoftgoal 228

B.4 Código-fonte da Classe DecomposedSoftgoal 233

B.5 Código-fonte da Enumeração DecompositionType 235

B.6 Código-fonte da Classe ImpactSoftgoal 235

B.7 Código-fonte da Classe Task 237

B.8 Código-fonte da Classe Contribution 239

B.9 Código-fonte da Enumeração ContributionType 240

Apêndice C. Código-Fonte das Principais Situações do

Estudo de Caso Lattes-Scholar 242

C.1 Situação "Buscar o Pesquisador" 242

C.1.1 Código-fonte do ADF do agente Buscador buscador.agent.xml 242 
C.1.2 Código-fonte da Classe ExecuteRPRequest.java

C.1.3 Código-fonte do ADF da Capacidade Google

Google.capability.xml 248

C.1.4 Código-fonte da Classe BuscarPesquisadoresNoGoogle.java $\quad 250$

C.2 Situação "Decidir entre Utilizar o Agente Fornecedor de Citações Local ou um Remoto"

C.2.1 Código-fonte do ADF do Agente Fornecedor de Citações FornecedorDeCitações.agent.xml 256

C.2.2 Código-fonte da Classe SetupAgent.java 260

C.2.3 Código-fonte da Classe

RequisitarONumeroDeCitacoesParaOGoogleScholar.java 263

C.2.4 Código-fonte da Classe DelegarAMetaAUmAgenteRemoto.java 264

C.3 Código-fonte para a Situação "Obter as Citações da Publicação no Google Scholar"

C.3.1 Código-fonte do ADF do Agente Fornecedor de Citações FornecedorDeCitacoes.agent.xml 267

C.3.2 Código-fonte da Classe ExecuteRPRequest.java 269

C.3.3 Código-fonte do ADF da Capacidade Scholar

Scholar.capability.xml 272

C.3.4 Código-fonte da Classe ObterAsCitacoesNoGoogleScholar.java 275

C.3.5 Código-fonte da Classe

RecuperarAPaginaDaPublicacaoNoGoogle Scholar.java $\quad 277$

C.3.6 Código-fonte da Página lattesscholar.xhtml 


\section{Lista de Figuras}

Figura 2.1 - Um modelo i* 27

Figura 2.2 - Um exemplo de um SIG 28

Figura 2.3 - O SIG de Transparência, traduzido de (Leite e Cappelli 2010) 29

Figura 2.4 - Visão Geral da Arquitetura intra-agente utilizada no JADEX, extraída de (Pokahr e Braubach 2007) 33

Figura 2.5 - O schema XML de um agente Jadex, extraído de (JADEX 2007a) 33

Figura 2.6 - Exemplo de um plano que escolhe um movimento entre vários possíveis, extraído de (JADEX 2007a) 34

Figura 2.7 - Um exemplo da notação gráfica da linguagem ACE 36

Figura 3.1 - Relações entre as abstrações do framework $\mathrm{i}^{*}$ e do modelo BDI 41

Figura 3.2 - Relações entre as abstrações do modelo BDI e código JADEX 43

Figura 3.3 - Aplicação das heurísticas de desenho no modelo arquitetural parcial do Lattes-Scholar $\quad 48$

Figura 3.4 - Trechos de código que podem ser obtidos a partir dos cenários $\quad 52$

Figura 4.1 - Os graus de satisfação das metas flexíveis vistos como uma variável nebulosa.

Figura 4.2 - Duas tarefas contribuindo para uma meta 62

Figura 4.3 - Habilitando o agente com a capacidade da máquina de raciocínio qualitativa.

Figura 4.4 - A variável nebulosa (domínio e conjuntos nebulosos) de uma meta flexível

Figura 4.5 - Código parcial para a classe "Task" 68

Figura 4.6 - Código parcial para a classe "Contribution" 68

Figura 4.7 - Código de geração de regras nebulosas para o tipo de contribuição "help".

Figura 4.8 - Metas flexíveis, contribuições e tarefas como crenças dos agentes 69

Figura 4.9 - Inicialização de uma tarefa e sua contribição no setup do agente 69

Figura 4.10 - Código parcial da classe abstrata "Softgoal" 69

Figura 4.11 - Estrutura meios-fim do Lattes-Scholar $\quad 72$

Figura 4.12 - Estrutura meios-fim do Sistema de Iluminação Inteligente 83 
Figura 5.1 - As quatro estruturas $i^{*}$ recorrentes representadas como proposições na linguagem ACE 92

Figura 5.2 - Padrão de requisitos "Objetivos de Transparência de Software”, extraído do CTS (CTS 2011).

Figura 5.3 - As três estruturas SIGs recorrentes representadas como proposições na linguagem ACE

Figura 5.4 - Os late-requirements do Lattes-Scholar modelados no framework $\mathrm{i}^{*}$

Figura 5.5 - A estrutura i* meios-fim da Figura 5.4 traduzida para a linguagem ACE

Figura 5.6 - A evolução do Grafo D de D(0) até D(1) 100

Figura 5.7 - Grafo resultante $\mathrm{D}(2)=\mathrm{D}(3)=\mathrm{D}(\mathrm{N}) \quad 101$

Figura 5.8 - Um SIG parcial de Transparência 102

Figura 5.9 - Um conflito (C1) entre o RNF Validade (i $(n f r 9))$ e a tarefa $t l(\mathrm{i}(t l))$

Figura 5.10 - $\mathrm{O}$ argumento $p 2$ de um interessado a favor de uma estrutura meios-fim alternativa 104

Figura 5.11 - Argumentos ( $p 3$ e $p 4$ ) de dois interessados 105

Figura 5.12 - A regra de preferência ilustrando a preferência pela proposição $p 4 \quad 106$

Figura 5.13 - A estrutura i* meios-fim evoluída 106

Figura 5.14 - Um conflito (C8) entre o RNF Clareza (i(nfr7)) e a estrutura $i^{*}$ meios-fim $\left(\mathrm{I}_{\mathrm{ME} 3}\right)$

Figura 5.15 - Um argumento p7 de um interessado a favor da substituição da estrutura meios-fim $\mathrm{I}_{\mathrm{ME} 3}$ por uma estrutura meios-fim do tipo Ou-exclusivo

Figura 5.16 - A regra de preferência ilustrando a preferência pela proposição $p 7$

Figura 5.17 - A estrutura i* meios-fim evoluída

Figura 5.18 - O grafo ACE completo para o primeiro estudo de caso

Figura 5.19 - O grafo do léxico do SIG de Transparência

Figura 5.20 - O símbolo "iv-nfr8” enriquecido com elos para outros símbolos do LEL

Figura 6.1 - Estrutura em camadas do ITrace 
Figura 6.2 - Etnografia aplicada nas gravações audiovisuais de uma reunião 125

Figura 6.3 - Granularidade grossa para evolução de artefatos 125

Figura 6.4 - Modelo ITrace da decomposição do RNF Acurácia 127

Figura 6.5 - ITrace de um processo de avaliação de equipes de engenheiros de requisitos

Figura 6.6 - Explorando a funcionalidade de hipermídia do Flickr® (Flickr 2011b)

Figura 7.1 - O processo de desenvolvimento proposto modelado em SADT

Figura 7.2 - Storyboard produzido com quadros dos vídeos da segunda reunião

Figura 7.3 - O grafo ACE que representa a discussão sobre a disponibilização do Lattes-Scholar como uma aplicação Web

Figura 7.4 - Modelo ITrace da primeira reunião

Figura 7.5 - Modelo de Atores Estratégicos do Lattes-Scholar

Figura 7.6 - Modelo de Dependências Estratégicas do Lattes-Scholar para a situação "Recuperar citações"

Figura 7.7 - Modelo arquitetural em i* do SMA do Lattes-Scholar para a situação "Recuperar Citações"

Figura 7.8 - Modelo de Rationale Estratégico parcial do papel "Fornecedor de Citações" na situação "Recuperar Citações"

Figura 7.9 - A tarefa "Requisitar o número de citações para o Google

Scholar" detalhada como um cenário

Figura 7.10 - A especificação BDI do papel "Fornecedor de Citações" como um agente não executável

Figura 7.11 - ADF da capacidade "FornecedorDeCitacoes", produzido pelas heurísticas transformacionais de implementação

Figura 7.12 - Crenças definidas na capacidade "FornecedorDeCitacoes"

Figura 7.13 - As metas da capacidade "FornecedorDeCitacoes"

Figura 7.14 - Os planos da capacidade "FornecedorDeCitacoes"

Figura 7.15 - O plano "RequisitarONumeroDeCitacoesParaOGoogle Scholar" anotado com cenários

Figura 7.16 - Implementando os episódios entre os comentários 


\section{Lista de Tabelas}

Tabela 2.1 - Operacionalizações e questões para a meta flexível folha Validade 30

Tabela 3.1 - Heurísticas transformacionais de desenho: do framework $\mathrm{i}^{*}$ para a especificação BDI

Tabela 3.2 - Heurísticas transformacionais de implementação: da especificação BDI para código JADEX

Tabela 4.1 - Regras de propagação do framework i* (ISTARWIKI 2011). 62

Tabela 4.2 - Situação inicial das metas flexíveis 73

Tabela 4.3 - Impactos atribuídos às tarefas na análise da primeira alternativa 73

Tabela 4.4 - Regras nebulosas executadas na análise da primeira alternativa 74

Tabela 4.5 - Impactos atribuídos às metas flexíveis caso a primeira alternativa seja selecionada 75

Tabela 4.6 - Impactos atribuídos às tarefas na análise da segunda alternativa 75

Tabela 4.7 - Regras nebulosas executadas na análise da segunda alternativa 76

Tabela 4.8 - Impactos atribuídos às metas flexíveis caso a primeira alternativa seja selecionada 76

Tabela 4.9 - Situação atual das metas flexíveis 77

Tabela 4.10 - Impactos atribuídos às tarefas na análise da primeira alternativa 78

Tabela 4.11 - Regras nebulosas executadas na análise da primeira alternativa 79

Tabela 4.12 - Conjunto de impactos resultante da seleção da primeira $\begin{array}{ll}\text { alternativa } & 80\end{array}$

Tabela 4.13 - Impactos atribuídos às tarefas na análise da segunda alternativa 80

Tabela 4.14 - Regras nebulosas executadas na análise da segunda alternativa 81

Tabela 4.15 - Conjunto de impactos resultante da seleção da segunda alternativa $\quad 82$

Tabela 5.1 - A informação do símbolo "iv-p4” 112

Tabela 5.2 - A informação do símbolo "ir-t4" 112

Tabela 5.3 - A informação do símbolo "ir-me3" 112 


\section{Lista de Abreviaturas e Siglas}

AC - Acceptability Condition

ACE - Acceptability Evaluation

AIF - Argument Interchange Format

API - Application Program Interface

ADF - Agent Definition File

BDI - Belief Desire Intention

C\&L - Cenários e Léxico

CASE - Computer-Aided Software Engineering

CTS - Catálogo de Transparência de Software

DEP - Dependency

ER - Engenharia de Requisitos

FIPA - Foundation for Intelligent Physical Agents

GORE - Goal-Oriented Requirements Engineering

GQM - Goal-Question-Metric

GQO - Goal-Question-Operationalization

i $^{*}$ - iStar, iEstrela ou Intencionalidade Distribuída

IDE - Integrated Development Environment

JADE - Java Agent Development Environment

JADEX - Java Agent Development Environment eXtension

LEL - Léxico Estendido da Linguagem

ME - Means-End

NFR - Non-Functional Requirements

OME - Organization Modelling Environment

OP - Operationalization

RNF - Requisito Não-Funcional

SA - Strategic Actors

SADT - Structured Analysis and Design Technique

SD - Strategic Dependency

SIG - Softgoals Interdependency Graph 
SMA - Sistema Multi-Agentes

SR - Strategic Rationale

TROPOS - from trepō in Greek, which means "turning or adopting a new manner"

UML - Unified Modeling Language

URL - Uniform Resource Location

XML - eXtensible Markup Language 